\title{
A Netlogo and Matlab Hybrid Approach for Constructal Design of the Double-T Shaped Cavity by Means of Simulated Annealing
}

\author{
Gill V. Gonzales ${ }^{1}$ \\ Instituto Federal Sul-rio-grandense Campus Santana do Livramento, Rua Paul Harris, 410, Centro, \\ CEP 97574-360, Sant'Ana do Livramento - RS - Brazil \\ Elizaldo D. dos Santos ${ }^{2}$ \\ Diana F. Adamatti ${ }^{3}$ \\ Programa de Pós-Graduação em Modelagem Computacional, Universidade Federal do Rio Grande, \\ Av. Itália, km 8, CEP 96201-900, Rio Grande, RS, Brazil \\ Antônio J. Silva Neto ${ }^{4}$ \\ Instituto Politécnico, Universidade do Estado do Rio de Janeiro, Rua Bonfim, 25, Vila Amélia, \\ Nova Friburgo, RJ, CEP 28.625-570. Brazil
}

\begin{abstract}
This paper describes the use of the NetLogo environment to perform the Constructal Design of a Double-T shaped Cavity with the stochastic algorithm Simulated Annealing. The algorithm is used in the geometric optimization of a heat transfer problem, which consists in a solid body with an internal uniform heat generation, which is cooled by a cavity that is maintained at a minimal temperature. The other surfaces are kept with an adiabatic condition. The objective is to minimize the maximum excess of temperature in the solid domain through geometric optimization of the isothermal double-T shaped cavity.
\end{abstract}

Keywords. Constructal Design, Simulated Annealing, Geometric Optimization, Heat Transfer

\section{Introduction}

The Constructal Theory is based on the view that the shapes and structures that emerge in nature are not due to a random cause, but a design ruled by a physical phenomenon. According to Bejan [1], there is a law that governs the designs in nature, the Constructal Law. The Constructal Law defines that "For a finite-size flow system to persist in time (to live), its configuration must evolve in such a way that provides easier access to the currents that flow through it". The application of this theory in engineering

\footnotetext{
${ }^{1}$ gillgonzales@ifsul.edu.br

${ }^{2}$ elizaldodossantos@gmail.com

3 dianaada@gmail.com

${ }^{4}$ ajsneto@iprj.uerj.br
} 
consists on the Constructal Design (CD) method. With this method it is possible to define constraints and objectives of an optimization problem according to the Constructal Theory. However, the CD is not an optimization method, but the method that helps to find the best distribution of imperfections in any flow system.

The study of cavity was firstly proposed by [3], when it was investigated the first and second elementary construction of the C-shaped and T-shaped cavities. The CD method is used to define constraints and degrees of freedom and the Exhaustive Search (ES) to find the optimal geometry recommendation. In [4] the H-shaped cavity was optimized and the achieved results showed that more complex cavity tends to have a best thermal performance. Some examples can be seen in complex and multi cavities studies [7,13]. However, the more complex is the cavity geometry, more degrees of freedom must be optimized. Then more computational effort is needed in the optimization process. In this case, the ES, where all possibilities are evaluated to achieve the optimal geometry, is impossible to be employed. Recently [5,7] the ES has been replaced by meta-heuristic strategies in the optimization process to find the optimal shapes. The double-T shaped cavity was proposed by [5] employing the Simulated Annealing to the geometric optimization.

In this paper it is performed the geometric optimization of the isothermal double- $T$ shaped cavity that influences the thermal performance of fins array. The double-T shaped cavity has five degrees of freedom (DOFs). In this study, four DOFs are optimized through SA implemented in the NetLogo environment. The results obtained are compared with the results shown in [5], in order to validate the use of NetLogo approach in the Constructal Design problem. The numerical solver that evaluated the temperature fields is the PDE Tools of the MATLAB ${ }^{\circledR}$ environment. An integration with NetLogo was performed to run the SA algorithm. The MATLAB ${ }^{\circledR}$ solver is used to compute the objective function for the SA algorithm in NetLogo. This integration is also implemented in other works such as in [2]. In this work the integration between these two models frameworks is being analyzed as a first step to validate the employment of NetLogo with Constructal Design. The advantages of the NetLogo approach are the easy integration with the MATLAB ${ }^{\circledR}$ and graphical user interface building, as well as, the possibility to use the multi-agent models in the geometric optimization of heat transfer problems.

\section{Mathematical and Numerical Model}

Figure 1 shows the conducting body in the two-dimensional configuration, with the third dimension, with length $W$, perpendicular to the plane of the figure. The solid domain is represented by the gray region in Fig. 1, which has a constant and uniform internal heat generation at the volumetric rate given by $q^{\prime \prime \prime}\left(W m^{-3}\right)$. The solid has a constant thermal conductivity k. The outer surfaces of the solid are perfectly insulated, corresponding to adiabatic conditions. In this case, the heat can only be removed through the double- $T$ shaped cavity, which is kept at a minimum temperature $\left(\theta_{\text {min }}\right)$. The minimal temperature of the cavity may be kept with the flow of refrigerant fluid through the cavity, changing phase at low temperature flowing. For the sake of simplicity, the heat transfer coefficient on the cavity wall is assumed so large that the convective resistance can be neglected in 
comparison to the solid conduction resistance.

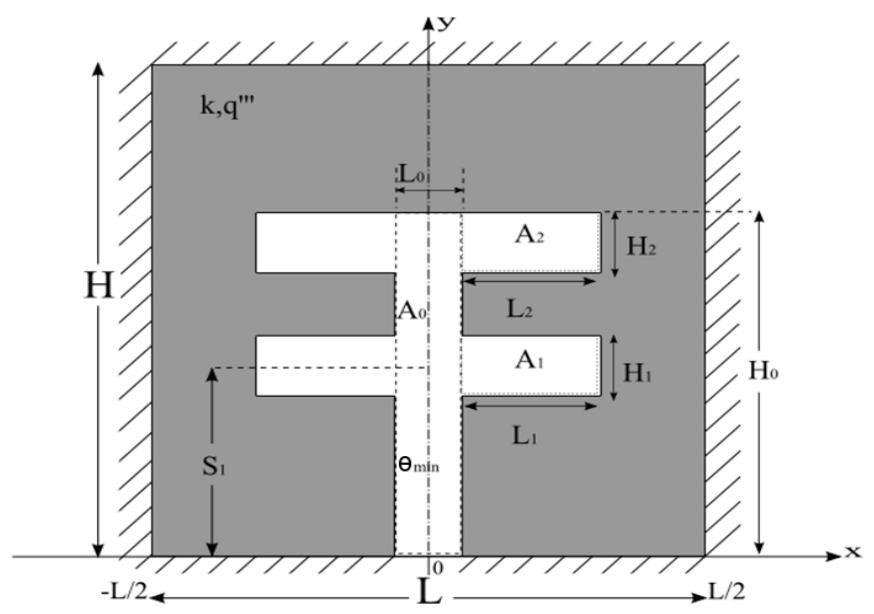

Figure 1: Computational domain of Double-T shaped cavity.

The objective of the analysis is to determine the optimal geometry $\left(H / L, H_{0} / L_{0}\right.$, $H_{1} / L_{1}, H_{2} / L_{2}$ and $\left.S_{1} / H_{0}\right)$ that is characterized by the minimum global thermal resistance $\left(\theta_{\max }-\theta_{\min }\right) /\left(q^{\prime \prime \prime} A\right)$. According to $\mathrm{CD}$ this optimization can be subjected to the constraints of the total area and cavity area, represented respectively by following equations:

$$
\begin{gathered}
A=H L \\
A_{c}=A_{0}+2 A_{1}+2 A_{2}
\end{gathered}
$$

The fraction of the cavity area in relation to total area is given by:

$$
\phi_{c}=A_{c} / A
$$

For the determination of the temperature field in the solid domain, it is necessary to solve the heat conduction equation given by:

$$
\frac{\partial \theta}{\partial \tilde{x}^{2}}+\frac{\partial \theta}{\partial \tilde{y}^{2}}+1=0
$$

where the dimensionless variables are:

$$
\begin{gathered}
\tilde{\theta}=\frac{\theta-\theta_{\text {min }}}{q^{\prime \prime \prime} \cdot \frac{A}{k}} \\
\tilde{x}, \tilde{y}, \tilde{H}_{0}, \tilde{H}_{1}, \tilde{H}_{2}, \tilde{L}_{0}, \tilde{L}_{1}, \tilde{L}_{2}, \tilde{H}, \tilde{L}, \tilde{S}_{1}=\frac{x, y, H_{0}, H_{1}, H_{2}, L_{0}, L_{1}, L_{2}, H, L, S_{1}}{A^{1 / 2}}
\end{gathered}
$$

For the sake of brevity, the equations of boundary conditions of null flux in the solid outer surfaces, as well as, the equations of boundary conditions of minimal temperature in the cavity wall can be seen in the study of [5]. 
The dimensionless form of the Eqs. 1-2 are represented by the following equations:

$$
\begin{gathered}
1=\tilde{H} \tilde{L} \\
\phi_{c}=\tilde{H}_{0} \tilde{L}_{0}+2 \phi_{1}+2 \phi_{2} \\
\phi_{1}=\tilde{H}_{1} \tilde{L}_{1} \\
\phi_{2}=\tilde{H}_{2} \tilde{L}_{2}
\end{gathered}
$$

The aim is to minimize the maximal excess temperature represented by the following equation:

$$
\tilde{\theta}_{\max }=\frac{\theta_{\max }-\theta_{\min }}{q^{\prime \prime \prime} \cdot \frac{A}{k}}
$$

The determination of $\tilde{\theta}_{\max }$ it is needed to optimize the five degrees of freedom $(H / L$, $H_{0} / L_{0}, H_{1} / L_{1}, H_{2} / L_{2}$ and $\left.S_{1} / H_{0}\right)$ submitted at the corresponding constraints of the cavity area $\left(\phi_{c}, \phi_{1}\right.$ and $\left.\phi_{2}\right)$ and the total solid area. In this paper is optimized just four $\operatorname{DOF}\left(H_{0} / L_{0}, H_{1} / L_{1}, H_{2} / L_{2}\right.$ and $\left.S_{1} / H_{0}\right)$ keeping fixed $H / L=1$ and $A=1, \phi_{c}=0.1 ; \phi_{1}$ $=0.015 ; \phi_{2}=0.015$.

The function represented by Eq. 11 is determined numerically by solving Eq. 4 for the temperature field in every assumed configurations $\left(H / L, H_{0} / L_{0}, H_{1} / L_{1}, H_{2} / L_{2}\right.$ and $S_{1} / H_{0}$ ) and calculating $\tilde{\theta}_{\max }$ to see whether its value may be minimized by varying the configuration. The numerical solution is performed with the Finite Element Method (FEM) see Ref. [11], based on linear triangular elements, developed in the The MATLAB ${ }^{\circledR}$ environment, with the PDE (partial-differential-equations) toolbox [13]. The grid was non-uniform in both $\mathrm{x}$ and $\mathrm{y}$ directions, and varied from one geometry to the next. The appropriate mesh size was determined by successive refinements (h-adaptively), increasing the number of elements four times from one current mesh size to the next one. For the sake of brevity, the grid independence test can be seen in [5].

\section{Geometric Optimization}

The geometric optimization is conducted by the CD method that determines the objective and constraints of the problem. With the CD method, the constraints and degrees of freedom of the problem are defined, as well as the search space for the optimization process. The search of the optimal geometry is performed by an optimization method. In this paper, the SA algorithm is applied. The SA is implemented in the NetLogo programming language, and run in this environment. The objective function, represented by Eq. 11 , that must be minimized, is compute with the PDE toolbox of MATLAB ${ }^{\circledR}$. Therefore, it is needed an integration between this two platforms. The integration of NetLogo and MATLAB ${ }^{\circledR}$ was proposed by [2] with the MatNet extension for NetLogo environment. With MatNet extension it is possible execute in NetLogo a function developed in MATLAB ${ }^{\circledR}$ and share information between the two software.

The Constructal Design determines that the optimization process must be submitted to constraints that jointly with the objective function define the design of shape and 
structure of the system. For the sake of brevity, the equations that show the variables definition as functions of the DOFs can be seen in Gonzales et al. [5]. In order to validate the NetLogo approach only four DOFs are analyzed and the results are compared to the recent literature [5]. Therefore, the results achieved here may help future studies on complete optimization and the influence of the constraints over the optimal geometry of the Double-T shaped cavity.

The SA algorithm was developed in the NetLogo programming language according to the classical algorithm [6] and the MATLAB ${ }^{\circledR}$ implementation with parameters of Reannealing and StallIterLimited [8]. The Cooling Schedule (CS) is the main parameter of the SA algorithm, so in this work are employed the hybrid parameter (BoltxExp) studied in recent works [5]. The other parameters of SA algorithm are fixed for all versions studied here (Reannealing and StarIterLimited). Therefore, twenty rounds of the SA algorithm in NetLogo environment are performed and their results are stored for a generation of a database. These results are compared with those of Gonzales et al. [5], which were obtained with SA with the same parameters.

\section{Results and Discussion}

To validate the results of the SA in NetLogo it is performed a comparison with the results of the SA algorithm developed in $\mathrm{MATLAB}^{\circledR}$ for the same problem [5]. To perform this comparison, it is needed a statistical test over a sample of results of the various runs of the algorithm. In this work, the results of twenty rounds of the SA in NetLogo are performed and stored. The same number of the runs are executed in Ref. [5].

The statistical test performed is the Kruskal-Wallis test for non-parametric data. The Software $\mathrm{R}$ is used to statistical analysis with the non-parametric test [10]. Figure 2 shows a boxplot with data of two algorithm called samat and sanet. The samat represents the SA implemented in MATLAB ${ }^{\circledR}$, Ref. [5]. The sanet is the SA developed in NetLogo for this work. In Fig. 2 it is also possible to observe the classification of two algorithms in the same group $a$. Therefore, according to the results of the Kruskal-Wallis comparison the SA algorithm developed in the NetLogo environment does not have a statistically significant difference between the results of the SA algorithm implemented in MATLAB ${ }^{\circledR}$ Ref. [5].

The main advantages of NetLogo in comparison with MATLAB ${ }^{\circledR}$ environment are the easy building of the user interface and the direct integration between the interface components and algorithm parameters. With the interface, the parameters of the SA algorithm can be changed without change source code lines and it is possible easily follow the progress and variables state of the SA algorithm with the monitor components of the NetLogo. The MATLAB ${ }^{\circledR}$ environment also have a tool to design interface, but the integration is not automatic between components and variables. Then, researcher must develop this integration leading to a requirement of more knowledge about the graphical user interface development.

Concerning the performance of the two approaches, the NetLogo algorithm is slower than MATLAB $^{\circledR}$ because the integration is not instantaneous. The test was executed in the same machine with the same hardware configuration and with controlled process. 
MATLAB ${ }^{\circledR}$ version was approximately $36 \%$ faster than NetLogo version. The mean time for MATLAB ${ }^{\circledR}$ was $186 \mathrm{~s}$ while the mean time for NetLogo version was 295s. This occurred mainly because the NetMat extension is more one layer of software in the optimization process.

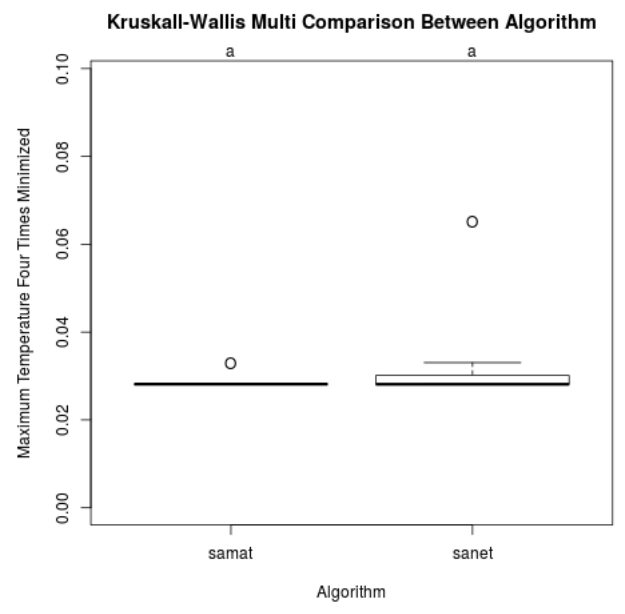

Figure 2: Comparison through by Kruskall-Wallis statistical test.

\section{Conclusions}

In this work it was performed a comparison between the results obtained with the same version and configuration parameters of the SA algorithm for the geometric optimization of Double-T Shaped Cavity developed on different programming language, MATLAB ${ }^{\circledR}$ and NetLogo. The results of the statistical test have shown that the distribution of the algorithms results does not have a statistically significant difference, and that the programming environment does not influences the results. The only disadvantage is the slow performance of NetLogo compared to MATLAB ${ }^{\circledR}$. In spite of this fact, this difference does not disregard the use of the NetLogo approach. Futures studies can be applied in the hybrid environment with MATLAB ${ }^{\circledR}$ and NetLogo. With the NetLogo environment, multi-agents models, as population algorithms, can be performed combined with Constructal Design to geometric optimization of the heat transfer problem.

\section{Acknowledgements}

G. V. Gonzales acknowledges IF-SUL and PPGMC-FURG by support. E. D. dos Santos is sponsored by CNPq. Antônio J. Silva Neto acknowledges the financial support provided by FAPERJ, CNPq and CAPES. 


\section{References}

[1] A. Bejan, Constructal-theory Network of Conducting Path for Cooling a Heat Generating Volume. Int. J. Heat Mass Transfer, vol. 40, n. 4, pp.799-816, 1997.

[2] M. B. Biggs,J. A. Papin, Novel Multiscale Modeling Tool Applied to Pseudomonas Aeruginosa Biofilm Formation. PLoS ONE, 8(10):e78011, 2013.

[3] C. Biserni, L. A. O. Rocha, A. Bejan, Inverted Fins: Geometric Optimization of the Intrusion Into a Conducting Wall. Int. J. Heat and Mass Transfer, 47, pp. 2577-2586, 2004 .

[4] C. Biserni, L. A. O. Rocha, G. Stanescu, E. Lorenzini, Constructal H-shaped cavities according to Bejan?s Theory. Int. J. Heat and Mass Transfer, 50, pp. 2132-2138, 2007.

[5] G. V. Gonzales, E. D. Dos Santos, L. A. Isoldi, E. da S. D. Estrada,L. A. O. Rocha, Constructal Design of Isothermal Double-T Shaped Cavity By Means of Simulated Annealing. In Proceedings of the XXXVI Iberian Latin-American Congress on Computational Methods in Engineering, Rio de Janeiro, RJ, Brazil, 2015.

[6] S. Kirkpatrick, C. D. Gelatt, M. P. Vecchi, M. P., Optimization by Simulated Annealing. Science, New Series., v. 220, No 4598, pp 671-680, 1983.

[7] G. Lorenzini, C. Biserni, E. da S. D. Estrada, E. D. Dos Santos, L. A. Isoldi, L. A. O. Rocha, Genetic Algorithm Applied to Geometric Optimization of Isothermal Y-Shaped Cavities. Journal of Electronic Packaging, vol 136, p. 031011-031011-9, 2014.

[8] MATLAB User?s Guide, Version 6.0.088, Release 12, The Mathworks Inc., Natick, MA, 2000.

[9] N. Metropolis, A. W. Rosenbluth, M. N. Rosenbluth, A. H. Teller, E. Teller, Equation of State Calculations by Fast Computing Machines. The Journal of Chemical Physics., v 21, p 1088-1092, 1953.

[10] R Core Team: R: A language and environment for statistical computing. R Foundation for Statistical Computing, Vienna, http://www.R-project.org, 2015.

[11] J. N. Reddy, D. K. Gartling, The Finite Element Method in Heat Transfer and Fluid Dynamics. CRC, Boca Raton, Florida,USA, 1994.

[12] U. Wilensky, NetLogo, Evanston IL NU: Center for Connected Learning and Computer-Based Modeling, 1999.

[13] Z. H. Xie, L. G. Chen, F. R. Sun, Constructal Optimization of Twice Y-Shaped Assemblies of Fins by Taking Maximum Thermal Resistance Minimization as Objective. Sci. China Technol. Sci., v. 53, pp. 2756-2764, 2010. 